\title{
Downregulation of long non-coding RNA ZXF1 restricts cell survival by targeting miR-634-GRB2 in lung adenocarcinoma
}

\author{
Xubin Ren ${ }^{1 \#}$, Nie Xu2\#, Yunting Zhang ${ }^{3 \otimes}$ and Tao Wang ${ }^{3}$ \\ 'Department of Respiratory and Critical Medicine, Chengdu First People's Hospital, Integrated TCM \& Western Medicine Hospital affiliated to \\ Chengdu University of TCM, Chengdu City, Sichuan Province, 610000, China; 2Department of Oncology, Chengdu First People's Hospital, Inte- \\ grated TCM \& Western Medicine Hospital affiliated to Chengdu University of TCM, Chengdu City, Sichuan Province, 610000, China; ${ }^{3}$ Department \\ of Respiratory and Critical Care Medicine, Renmin Hospital of Wuhan University, Wuhan City, Hubei Province, 430061, China
}

\begin{abstract}
Increasing evidence demonstrates that long non-coding RNAs (IncRNAs) play important regulatory roles in mediating initiation and progression of lung adenocarcinoma (LA), which is one of the most lethal in humans. A previous study reported that IncRNAZXF1 was dysregulated in LA and enhanced expression of ZXF1 promoted the invasion and metastasis in LA. However, the effect of ZXF1 on LA progression and its underlying mechanisms were not thoroughly investigated. In our in vitro experiments, qRT-PCR revealed that the expression level of ZXF1 in LA tissues and tumor cells were significantly higher than that in adjacent normal tissues and normal cells. Furthermore, bioinformatics analysis, luciferase reporter assay, western blot and RNA immunoprecipitation (RIP) assay showed that ZXF1 could directly interact with miR-634, which targets GRB2. Therefore, we propose that ZXF1 could function as an oncogene partly by sponging miR634 and therefore regulating GRB2 expression in LA. Overall, this study demonstrated, for the first time, that the IncRNA ZXF1/miR-634/GRB2 axis plays crucial roles in modulating LA progression. Moreover, IncRNA ZXF1 might potentially improve LA prognosis and serve as a therapeutic target for the treatment of LA.
\end{abstract}

Key words: Long non-coding RNA, ZXF1, lung adenocarcinoma, miR634, GRB2

Received: 29 September, 2019; revised: 10 December, 2019; accepted: 10 January, 2020; available on-line: 11 March, 2020

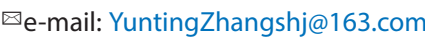

\#These authors contributed equally to the work

Abbreviations: ceRNA, competing endogenous RNAs; GBR2, growth factor receptor-bound protein 2; GRB2-mut, mutant type GRB2; GRB2-wt, wild type GRB2; ISH, in situ hybridization; LA, lung adenocarcinoma; IncRNAs, long non-coding RNAs; miR-634 inh, miR-634 inhibitor; miRNAs, MicroRNAs; NC inh, NC inhibitor; NSCLC, non-small cell lung carcinomas; RIP, RNA immunoprecipitation; RISC, RNA induced silencing complex; SCLC, small-cell lung carcinomas; ZXF1-mut, mutant type ZXF1; ZXF1-wt, wild type ZXF1

\section{INTRODUCTION}

Lung cancer is one of the most common public health problems and the leading cause of cancer-related mortality worldwide. In 2018, the most recent statistics from the American Cancer Society showed that lung cancer accounts for about $14 \%$ of new cancers in men and $13 \%$ of new cancers in women in the United States (Siegel et al., 2018). Lung cancers are routinely classified into small-cell lung carcinomas (SCLC) and nonsmall cell lung carcinomas (NSCLC). More than $80 \%$ of lung cancers are classified as non-small cell lung cancer
(NSCLC), and lung adenocarcinoma (LA) is the most common form of NSCLC (Zappa \& Mousa, 2016). Traditional treatments including surgery, chemotherapy, radiotherapy and blockade of epidermal growth factor receptor do not result in satisfying prognosis and the overall survival rate of LA is still very low (Wakeam et al., 2017). Therefore, it is necessary to thoroughly study the molecular pathogenesis of LA and explore the effective diagnostic biomarkers to improve clinical outcomes for the patients.

Long non-coding RNAs (lncRNAs) are one type of non-coding RNA molecules that are longer than 200 bp and lack protein-coding capability. Many biological processes such as transcription, translation, and posttranscriptional modification are regulated by lncRNAs (Ponting et al., 2009; Ni et al., 2018). A number of studies showed that $\operatorname{lncRNAs}$ participate in the initiation and progression of human cancers such as breast cancer (Zhou et al., 2018), ovarian cancer (Nikpayam et al., 2017), and liver cancer (Dickson, 2016). In recent years, lncRNAs were demonstrated to play a key regulatory role in the development of LA (Zhan et al., 2017). The expression levels of lncRNAs are closely related to the survival and prognosis of cancer patients, but the specific molecular mechanisms have not been fully elucidated. Thus, exploring the biological functions and underlying mechanism of lncRNAs is a promising strategy to gain insight into the pathogenesis of LA and to develop novel LA diagnostic biomarkers and therapeutic targets. ZXF1 is located on human chromosome 10q23.31 and contains 3,985 nucleotides (Pan et al., 2017). A previous study reported that $\operatorname{lncRNA}$ ZXF1 was up-regulated in LA patients (Zhang et al., 2014). However, the underlying molecular mechanisms and the effect of lncRNA ZXF1 on the progression of LA were not thoroughly explored.

MicroRNAs (miRNAs) are short non-coding RNA molecules that are $21-25 \mathrm{bp}$ long. They participate in the initiation and progression of cancers including lung cancer (Bartel, 2004). miR-634, a subunit of miR-634 family, plays an important role in regulating tumor initiation, invasion and metastasis in esophageal carcinoma cells (Fujiwara et al., 2015), cervical cancer cells (Cong et al., 2016), and hepatocellular carcinoma cells (Zhang et al., 2016). Although the previous studies revealed the important role of miR-634 in lung cancer, the molecular mechanisms of miR-634 in LA still need to be further explored.

Growth factor receptor-bound protein 2 (GBR2) links activated epidermal growth factor receptor tyrosine kinase to downstream kinases ERK1/2 by binding to 
specific phosphotyrosine-containing and proline-rich sequence motifs (Lowenstein et al., 1992). It was reported that GRB2 is highly expressed in a variety of cancers (breast cancer, gastric cancer and esophageal squamous cell carcinoma, etc.). It acts as an important tumor-promoting factor and is associated with metastasis and overall poor prognosis (Giubellino et al., 2008; Yu et al., 2009; Li et al., 2014). Several miRNAs were shown to target GRB2 to modulate cancer cell proliferation and migration at the post-transcriptional level. These miRNAs include miR-27b-3p (Chen et al., 2018), miR-433-3p (Shi et al., 2018), miR-329 (Wang et al., 2016) and miR-1258 (Jiang et al., 2018). However, whether the interaction between GRB2 and miR-634 is involved in the progression of LA is still not known.

Recent studies demonstrated that lncRNAs function as competing endogenous RNAs (ceRNA) by sponging miRNAs to regulate gene expression at the post-transcriptional level. In this study, we found that lncRNA ZXF1 acted as a decoy for miR-634 and GRB2 downregulation by miR-634 could be partially suppressed by lncRNA ZXF1 overexpression. Our study revealed a novel mechanism where lncRNA ZXF1 serves as a molecular sponge for miR-634 and regulates GRB2 expression level in LA progression.

\section{MATERIALS AND METHODS}

Collection of clinical specimens. The clinical LA tissues and matched adjacent normal lung tissues were collected from 60 patients with LA. These patients underwent surgical resection of the tumor. The normal lung tissues were $5 \mathrm{~cm}$ away from the edge of the tumor and no obvious tumor cells were found in these tissues by a tumor pathologist. All the cancer patients that participated in this research signed informed consents. The examination and the informed consents were approved by the Ethics Committee of Renmin Hospital of Wuhan University and conformed to the World Medical Association Declaration of Helsinki. The surgically removed tissue samples were frozen in liquid nitrogen promptly and stored at $-80^{\circ} \mathrm{C}$ until use.

Survival analysis. Data was collected from the Department of Oncology, Renmin Hospital of Wuhan University, Wuhan City, Hubei Province, China. It took about 5 years to collects the material, perform the experiment and collect data on the patients' survival.

Cell culture and transfections. Human lung epithelial cell line (BEAS-2B) and four LA cell lines CALU3, SPC-A1, NCIH1573 and A549 were purchased from the Chinese Academy of Sciences (Shanghai, China). All of the cells were cultured in Dulbecco's modified Eagle's medium (DMEM) supplemented with 10\% fetal bovine serum (FBS) and 1\% penicillin $(100 \mu \mathrm{g} / \mathrm{mL}) /$ streptomycin $(100 \mathrm{U} / \mathrm{mL})$ at $37^{\circ} \mathrm{C}$ in an incubator with $5 \%$ $\mathrm{CO}_{2}$. MiR-634 mimic, pcDNA3.1/ZXF1 plasmid were obtained from Gene-pharma (Shanghai, China). The siRNA sequences targeting GRB2 (si-GRB2) were purchased from RiboBio Co. Ltd (Guangzhou, China). All of the transfections in this project were performed with Lipofectamine 2000 reagent (Invitrogen, Carlsbad, CA, U.S.A.) according to the manufacturer's instructions.

RNA extraction and qRT-PCR. Total RNA was extracted from patients' tissues and cultured cell lines using Trizol reagent (Thermo Fisher Scientific, Inc., MA, USA) then reverse-transcribed to cDNA using the RevertAid RT Reverse Transcription kit (Thermo Fisher Scientific, Inc., MA, USA) according to the manufacturer's instruc- tions. The quantification of mRNA expression was performed by qRT-PCR using the Standard SYBR-Green RT-PCR kit (Takara Bio, Inc., Otsu, Japan) in accordance with the standard protocols and GAPDH was used as the control gene. All of the related miRNA reagents used in this study were purchased from RiboBio Co., Ltd (Guangzhou, China). MiR-634 mimic (5'-AACCAGCACCCCAACUUUGGACGGTATTCGCACTGGATACGACGAACT'T'T-3), miR-634 inhibitor (5-CACUACUUUUGUGUCCCACUU-3) were purchased from Guangzhou RiboBio Co., Ltd. (Guangzhou, China). MiR-634 primer was purchased from Shanghai GenePharma Co., Ltd. The relevant primer sequences were prepared (Sangon, Shanghai, China) as follows: ZXF1forward: 5'-GCCUGGUGGUAAAUAUGAAT'T-3' and ZXF1-reverse: 5'-UUCAUAUUUACCACCAGGCT'T-3'; GRB2-forward: 5'-ATTCCTGCGGGACATAGAACA-3' and GRB2-reverse: 5'-GGTGACATAATTGCGGGGAAAC-3'; GAPDH-forward: 5'-AGACACCATGGGGAAGGTGAA-3' and GAPDH-reverse: 5'-ATTGCT-

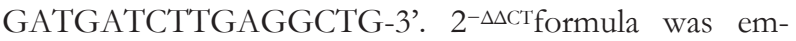
ployed to calculate the relative mRNA expression levels.

Cell proliferation assay. Cell proliferation was measured with CCK-8 assay according to the standard protocol. CCK-8 was purchased from Dojindo Chemi-

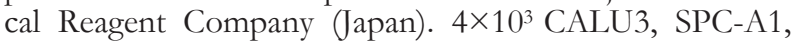
NCIH1573 and A549 cells were separately seeded in each well in 96-well plates. After incubated for $2 \mathrm{~h}$, a volume of $20 \mu \mathrm{L}$ CCK-8 was added to each well and incubated for $1 \mathrm{~h}$ at $37^{\circ} \mathrm{C}$. Viable cells were counted by measuring the OD (optical density) value at the wavelength of $450 \mathrm{~nm}$ using a microplate reader. All experiments were repeated 3 times and average results were used to draw the growth curves.

Cell cycle assay. Flow cytometry was performed for cell cycle analysis. Transfected cells (CALU3, SPC-A1, NCIH1573 and A549) were separately plated into 6-well plates at the density of $8 \times 10^{4}$ cells per well. After $48 \mathrm{~h}$ of incubation, the cells were harvested and resuspended in $500 \mu \mathrm{L}$ of buffer and then stained with PI for $30 \mathrm{~min}$. Finally, dead CALU3, SPC-A1, NCIH1573 and A549 cells thatstained by PI were analyzed. Cell cycle was analyzed using FACScan flow cytometer (Becton Dickinson). The cell cycle phases distribution was analyzed by ModFit LT software.

Western blot. RIPA Buffer (9800, Cell Signaling, Danvers, MA) was used to lyse and extract total proteins. After centrifugation, 12.5\% SDS-PAGE was used to separate the proteins. Subsequently, protein samples were transferred to the polyvinylidene fluoride (PVDF) membranes and blocked with $5 \%$ milk for $1 \mathrm{~h}$ at $37^{\circ} \mathrm{C}$. Next, the PVDF membranes were incubated with antibodies against GRB2, GAPDH, CyclinD1, and p21 (Santa Cruz Biotechnology, USA) at $37^{\circ} \mathrm{C}$ for $2 \mathrm{~h}$. Then, the PVDF membranes were washed and incubated with HRP-conjugated secondary antibodies for $1 \mathrm{~h}$ at room temperature. ECL detection reagents from Amersham Pharmacia Biotech (Tokyo, Japan) were used to detect the immunocomplexes.

Dual-luciferase reporter assay. To confirm the molecular interactions between ZXF1 and miR-634, as well as miR-634 and GRB2, luciferase reporter assays were performed in HEK-293 cells. The pmirGLO-ZXF1 wild type (ZXF1-wt) and pmirGLO-ZXF1 mutant (ZXF1mut), as well as pmirGLO-GRB2 wild type (GRB2-wt) and pmirGLO-GRB2 mutant (GRB2-mut), were designed and ordered from Sangon (Shanghai, China). $1.5 \times 10^{5}$ of HEK-293 cells were seeded into each well of 12 -well plates. Then, $30 \mathrm{nM}$ miR-634 mimic or miR- 
NC was co-transfected with 300 ng DNA (ZXF1-wt or ZXF1-mut as well as GRB2-wt and GRB2-mut) into HEK-293 cells using transfection reagent Lipofectamine 2000 (Invitrogen) in accordance with the manufacturer's instructions. $24 \mathrm{~h}$ after the transfection, the relative luciferase activity was assessed using Dual-Luciferase Reporter Assay System (Promega, Madison, WI, USA).

RNA immunoprecipitation (RIP). RNA immunoprecipitation (RIP) assay was performed with Magna RNA-binding protein immunoprecipitation kit (Millipore) according to the manufacturer's instructions. Briefly, the cell lysates of CALU3 and A549 cells were collected and incubated in RIP buffer containing magnetic beads conjugated to human anti-IgG antibody or anti-Ago2 antibody. Samples were incubated with Proteinase $\mathrm{K}$ and then immunoprecipitated RNA was isolated. In addition, the purified RNAs were extracted and analyzed by qRTPCR to demonstrate the presence of the binding targets. Anti-IgG antibody group denotes the negative control with IgG antibody. Anti-Ago2 antibody group denotes the experimental group with Ago2 antibody.

In vivo $\mathrm{Balb} / \mathrm{c}$ nude mice xenograft model. 4-week-old Balb/c nude mice (male) were purchased from Shanghai Experimental Animal Center (Shanghai, China). Next, $1 \times 10^{7}$ A549 cells in $0.2 \mathrm{~mL}$ PBS buffer were subcutaneously injected into each mouse to construct the Balb/c nude mice xenograft model. Then, the mice were injected intraperitoneally with sh-ZXF1 (equal to si-ZXF1) or sh-NC (equal to si-NC). After successfully obtaining a stable mouse model, the tumor size and volume [volume $\left(\mathrm{mm}^{3}\right)=$ width $^{2}\left(\mathrm{~mm}^{2}\right) \times$ length $(\mathrm{mm}) \div 2$ ] were measured every 3 days. Finally, after 24 days, the mice were sacrificed, and LA tumor tissues were isolated and used for further studies. This experiment was approved by the Ethics Committee of Renmin Hospital of Wuhan University.

Statistical analysis. All of the experiments performed in this project were repeated 3 times and the average values were shown. SPSS 19.0 statistical software was used to analyze the data and means \pm S.D. (standard deviation) were presented. The difference between the two groups was tested by independent sample $t$-test as well as Pearson's correlation coefficient analysis. A value of $p<0.05$ denotes statistically significant difference. $*_{\text {in- }}$ dicates $p<0.05$, **indicates $p<0.01$ and \#\#also indicates $p<0.01$.

\section{RESULTS}

\section{ZXFI is upregulated in LA patients}

To identify the correlations between ZXF1 and the pathogenesis of LA as well as patient survival, qRT-PCR and in situ hybridization (ISH) were used to analyze the expression of ZXF1 in isolated lung tumor tissues and adjacent normal tissues from 60 LA patients. Our data showed that when compared with the adjacent tissues, the expression of ZXF1 was significantly upregulated in the tumor tissues (Fig. 1A, $p<0.01$ ). In addition, LA cells had larger volume and different morphology and different nucleus to cytoplasm ratio compared with the normal cells (Fig. 1B). Subsequently, we analyzed the data using Kaplan-Meier plotter software and found that patients with low ZXF1 expression displayed higher survival rates, indicating that ZXF1 might serve as a tumor

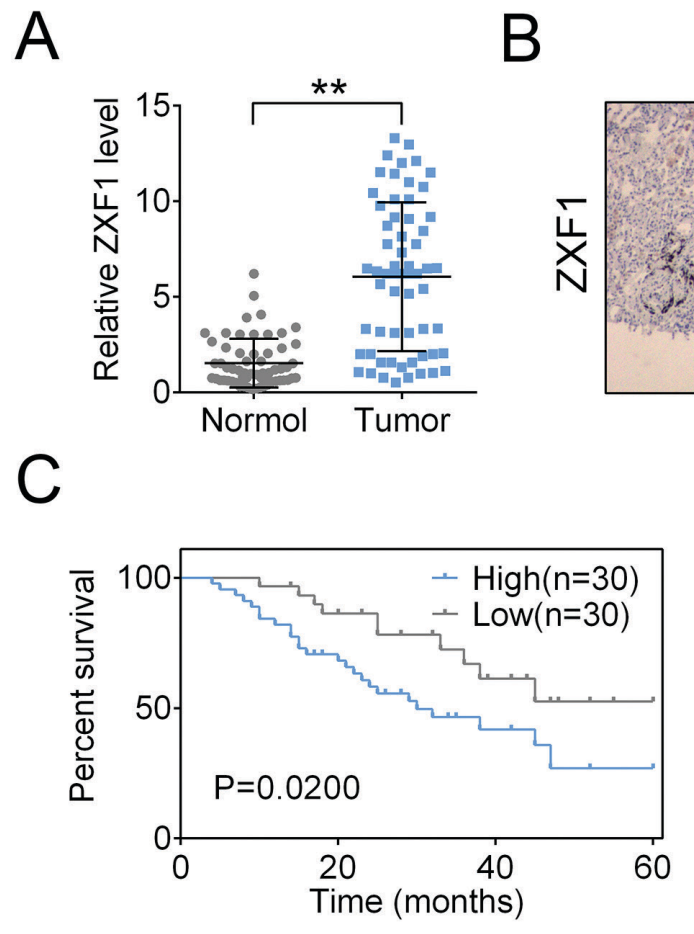

Normol
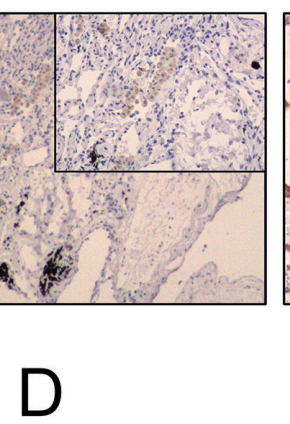

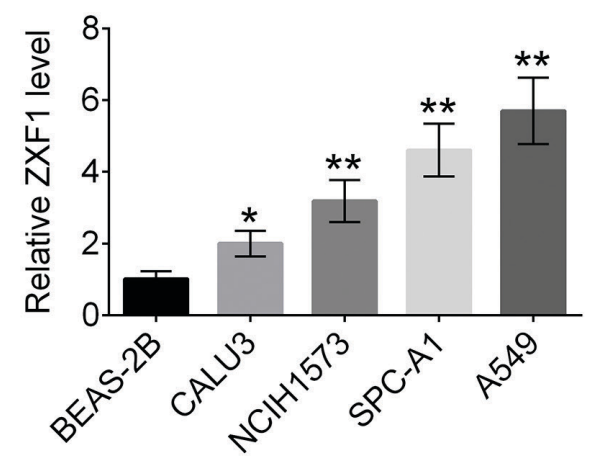

Figure 1. ZXF1 IncRNA was upregulated in lung adenocarcinoma tissues.

(A) The expression difference of ZXF1 in tumor tissues and normal tissues in 60 patients was analyzed by qRT-PCR. (B) ISH analysis of tumor tissues and matched adjacent normal tissues. (C) The overall survival analysis of ZXF1 expression in LA patients. (D) qRT-PCR analysis of the expression difference of ZXF1 in normal lung epithelial cell line (BEAS-2B) and four lung adenocarcinoma cell lines (CALU3, SPC-A1, NCIH1573 and A549). Results are presented as mean \pm S.D. $p$ values lower than 0.05 indicated statistical significance. ${ }^{*}$ Indicates $p<0.05$, **indicates $p<0.01$. 
A

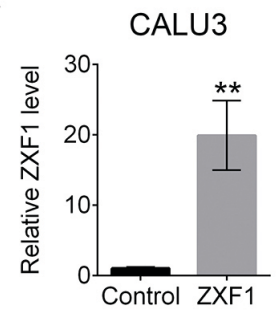

A549

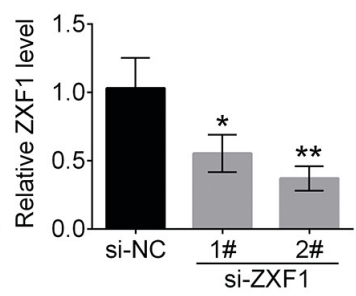

C

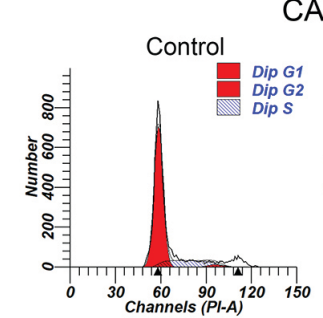

CALU3

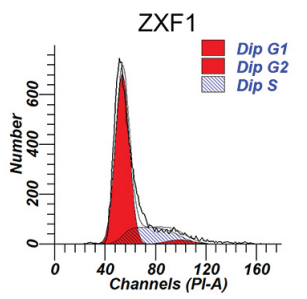

A549

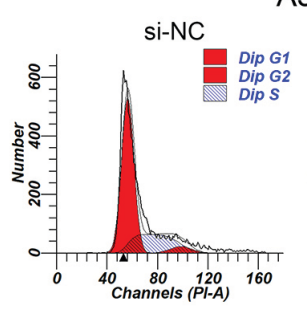

B

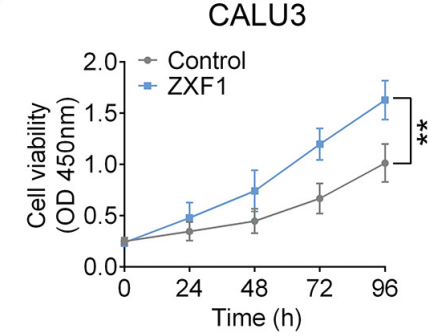

D

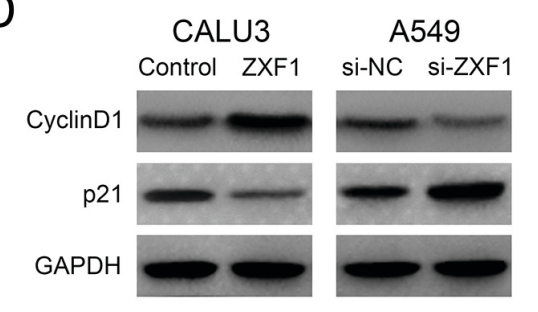

A549
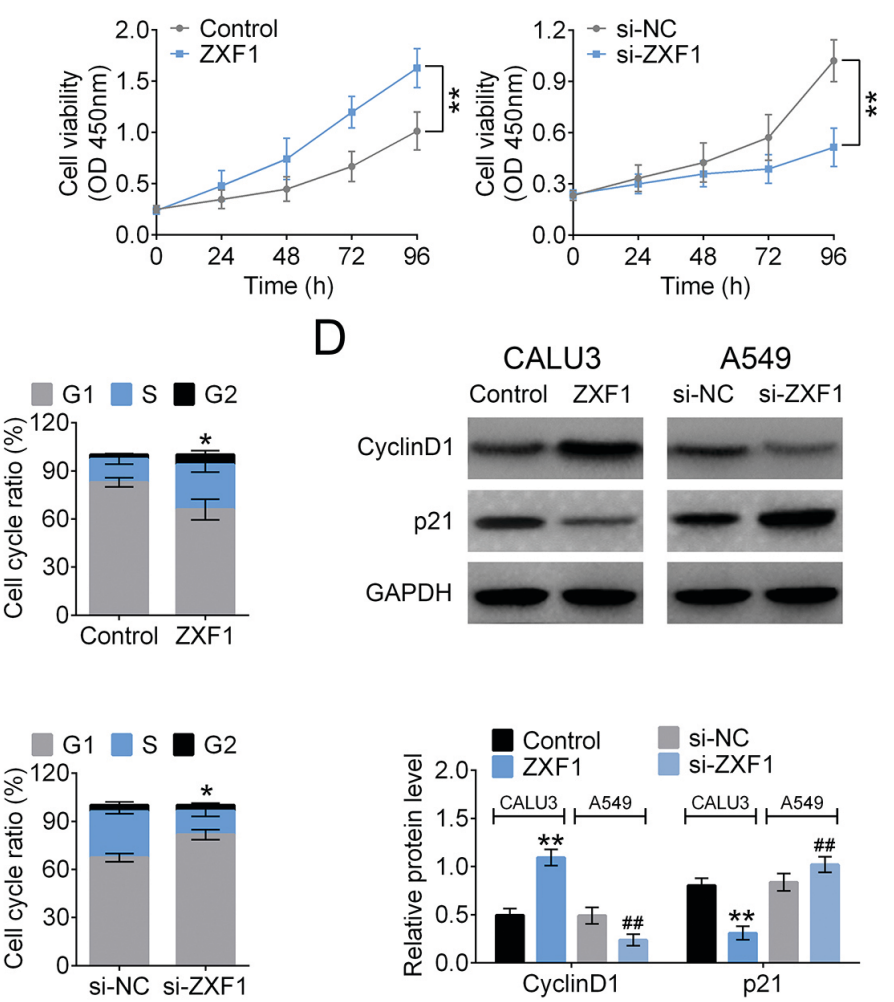

Figure 2. Inhibition of ZXF1 could suppress cell proliferation and promote apoptosis of lung adenocarcinoma cells in vitro.

(A) The mRNA expression levels of ZXF1 in CALU3 cells transfected with pcDNA3.1/ZXF1 as well as A549 cells transfected with si-NC or si-ZXF1 (1\#, 2\#) were quantified using qRT-PCR. (B) The cell proliferation of siRNA-transfected lung adenocarcinoma cells after 1, 2, 3 and 4 days were evaluated using CCK-8 assay. (C) The cell cycle phases distribution in siRNA-transfected lung adenocarcinoma cells were determined by flow cytometry analysis. (D) The protein expression levels of cell cycle regulatory protein Cyclin D1 and cyclin-dependent kinase inhibitor p21 in siRNA-transfected lung adenocarcinoma cells were analyzed by Western Blot. Dip denotes Diploid. Results are presented as mean \pm S.D. $p$ values lower than 0.05 indicated statistical significance. ${ }^{*}$ Indicates $p<0.05,{ }^{* *}$ indicates $p<0.01$.

promoter for patients with LA (Fig. 1C). We further evaluated the expression difference of ZXF1 in normal lung epithelial cell line (BEAS-2B) and four LA cell lines (CALU3, SPC-A1, NCIH1573 and A549) by qRT-PCR. Consistently with the results in tumor tissues, we found that the expression level of ZXF1 in normal lung epithelial cells was significantly lower than that in LA cell lines including CALU3, SPC-A1, NCIH1573 and A549 cells (Fig. 1D).

\section{Knockdown of ZXF1 suppresses LA cell proliferation in vitro}

To investigate the effect of ZXF1 on cell proliferation, we transfected CALU3 cells with pcDNA3.1/ZXF1 plasmid and A549 cells with si-NC or si-ZXF1 (1\#, 2\#). CALU3 cells transfected with pcDNA3.1/ZXF1 plasmid (ZXF1) displayed higher expression of ZXF1. We tested two si-ZXF1 and found that both lowered the expression of ZXF1 in A549 cells. Since si-ZXF1 (2\#) induced stronger reduction of ZXF1, we renamed it as si-ZXF1 and used it in the further experiments. CCK8 assay indicated that compared to control group, the cell proliferation rate in ZXF1-transfected CALU3 cells increased significantly $(p<0.05)$. On the other hand, the proliferation of si-ZXF1-transfected A549 cells was significantly inhibited $(p<0.01)$ (Fig. 2B). Therefore, it indicates that ZXF1 promotes cell proliferation in LA.
In addition, the effects of ZXF1 on the cell cycle were determined by flow cytometry analysis. Intriguingly, we found that ZXF1 overexpression led to an increase in the percentage of $\mathrm{S}$ phase and G2 phase and a decrease in the percentage of G1 phase in CALU3 cells. On the contrary, ZXF1 knock-down arrested A549 cells in G1 phase (Fig. 2C). This phenomenon showed that ZXF1 promotes cell proliferation, in accordance with the CCK8 assay results. Finally, we performed western blot to detect the protein levels of cell cycle regulatory protein Cyclin D1 and cyclin-dependent kinase inhibitor p21 in siRNA-transfected LA cells. The data demonstrated significant promotion of cell cycle by ZXF1 overexpression in vitro (Fig. 2D). Taken together, our results indicate that ZXF1 inhibition could suppress LA cells proliferation by arresting the cells in G1 phase of the cell cycle.

\section{ZXF1 directly interacts with miR-634 in LA cells}

Substantial evidence proved that lncRNAs work as a molecular sponge or a competing endogenous RNA to modulate miRNAs and thus functionally liberate other RNA transcripts. By predicting the potential miRNA binding sites in ZXF1 with bioinformatics software miRDB, we revealed that miR-634 may form complementary base pairing with ZXF1 (Fig. 3A). MiR-634 expression was increased significantly in miR-634 mimictransfected HEK-293 cells (Fig. 3B). In addition, the re- 
A

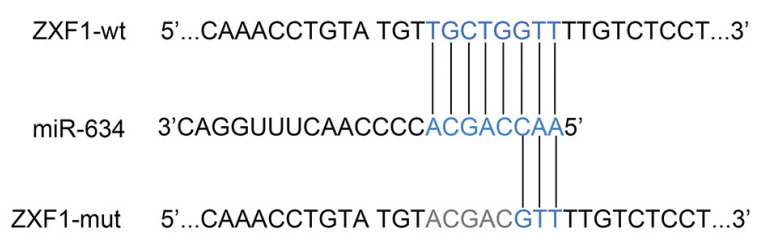

B

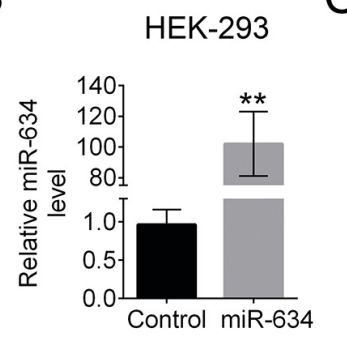

HEK-293

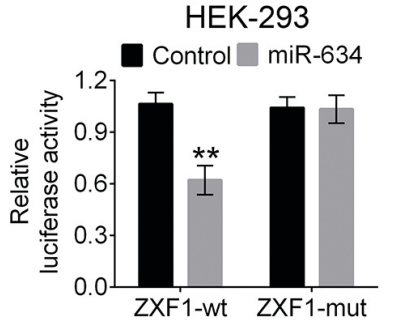

D

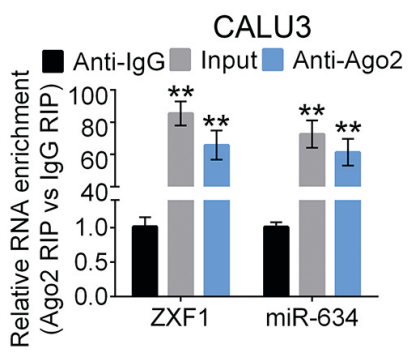

A549

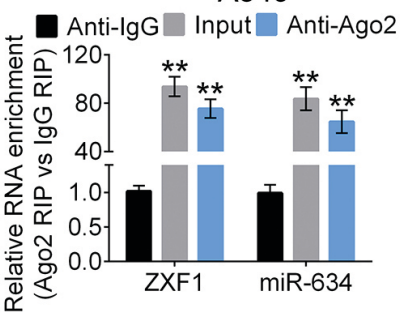

CALU3

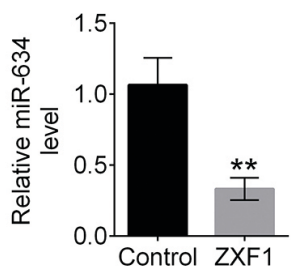

A549

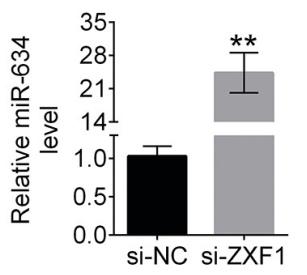

Figure 3. ZXF1 directly interacted with miR-634.

(A) The sequences of miR-634 binding sites complemented with ZXF1 3'-UTR (154-161). ZXF1-wt represents the entire 3'UTR sequences of wild type ZXF1, ZXF1-mut represents the mutated binding sites. (B) The mRNA expression of miR-634 in miR-634 mimic-transfected HEK-293 cells. (C) The binding affinity of miR-634 to ZXF1-wt or its mutant detected with luciferase reporter assay in HEK-293 cells. (D) The expression levels of ZXF1 and miR-634 were elevated in Ago2 immunoprecipitates in comparison to control IgG immunoprecipitates. (E) The expression of miR-634 in pcDNA3.1/ZXF1-transfected CALU3 cells as well as si-NC- / si-ZXF1-transfected A549 cells detected by qRT-PCR. Results are presented as mean \pm S.D. $p$ values lower than 0.05 indicated statistical significance. ${ }^{*}$ Indicates $p<0.05,{ }^{*}$ indicates $p<0.01$.

sults of luciferase reporter assays showed that miR-634 mimic significantly decreased the luciferase activity of wild type ZXF1 (ZXF1-wt), but not that of mutant type ZXF1 (ZXF1-mut) in HEK-293 cells. Thus, we believe that ZXF1-wt directly interacted with miR-634 (Fig. 3C). In order to identify whether ZXF1 and miR-643 were in the same RNA induced silencing complex (RISC), RNA immunoprecipitation (RIP) assay was performed both in CALU3 cells and A549 cells. Intriguingly, the data indicated that both ZXF1 and miR-643 were enriched in the anti-Ago2 group in comparison to the anti-IgG group (Fig. 3D). Finally, the expression of miR-634 in pcDNA3.1/ZXF1 (ZXF1)-transfected CALU3 cells as well as A549 cells transfected with si-NC or si-ZXF1 was evaluated by qRT-PCR. The results showed that overexpressing ZXF1 reduced miR-634 expression in CALU3 cells whereas knocking down ZXF1 upregulated miR634 in A549 cells (Fig. 3E). Therefore, the above results demonstrated that ZXF1 directly targets miR-634 in LA cells in vitro.

\section{ZXF1 upregulates GRB2 expression by sponging miR-634 in vitro}

Using bioinformatics software TargetScan we identified GRB2 as a downstream target gene of miR-634 (Fig. 4A). Luciferase reporter assays revealed that miR634 mimic significantly decreased the luciferase activity of wild type GRB2 (GRB2-wt), but not that of mutant type GRB2 (GRB2-mut) in HEK-293 cells (Fig. 4B). Thus, we believe that GRB2-wt directly interacts with miR-634. We then performed qRT-PCR to confirm the suppressive effect of miR-634 inhibitor (miR-634 inh) on the expression of miR-634 in CALU3 cells (Fig. 4C). Furthermore, Western Blot was used to examine the protein level of GRB2 in miR-634 mimic-transfected A549 cells and NC inhibitor (NC-inh)-/miR-634 inhibitor (miR634-inh)-transfected CALU3 cells (Fig. 4D). The results showed that overexpression of miR-634 downregulated GRB2 in A549 cells whereas knockdown of miR-634 upregulated GRB2 in GLU3 cells. To investigate how ZXF1 and miR-634 regulate the expression of GRB2, we used Western Blot and detected GRB2 in A549 cells transfected with si-ZXF1 alone or co-transfected with si-ZXF1 and miR-634 inhibitor. The data indicated that si-ZXF1 transfection could inhibit expression of GRB2. In addition, the inhibition was reversed by adding miR634 inhibitor (Fig. 4E). These results demonstrated that ZXF1could act as a sponge for miR-634 to upregulate the expression of GRB2 in vitro.

\section{ZXF1 increases LA cell survival by promoting GRB2 expression}

Accumulating evidence suggests that both ZXF1 and GRB2 function as important promotors of cancer initiation and progression. To investigate whether ZXF1 affected cell survival via GRB2 in LA cells, we transfected A549 cells with si-ZXF1-/GRB2 plasmid. qRT-CPR and Western Blot demonstrated that both the mRNA and protein level of GRB2 were significantly increased in GRB2 plasmid-transfected A549 cells (Fig. 5A, 5B). CCK-8 assay indicated that the cell proliferation rate in si-ZXF1-transfected A549 cells decreased significantly. However, after co-transfection of both GRB2 plasmid and si-ZXF1, the result was reversed $(p<0.05)$ (Fig. $5 \mathrm{C}$ ). In addition, the cell cycle was analyzed by flow cytometry. Remarkably, we found that knockdown of ZXF1 arrested A549 cells in G1 phase and overexpression of GRB2 in si-ZXF1 knockdown background resulted in an increase in the percentage of S phase and G2 phase, and a decrease in the percentage of G1 phase cells (Fig. 5D). This phenomenon showed that both ZXF1 and GRB2 could promote cell proliferation in LA, which is in accordance with the CCK-8 assay. Subsequently, we used Western Blot to analyze protein levels of Cyclin D1 and 
A

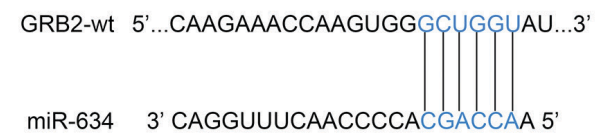

GRB2-mut 5'...CAAGAAACCAAGUGGCGACCAAU...3'

D

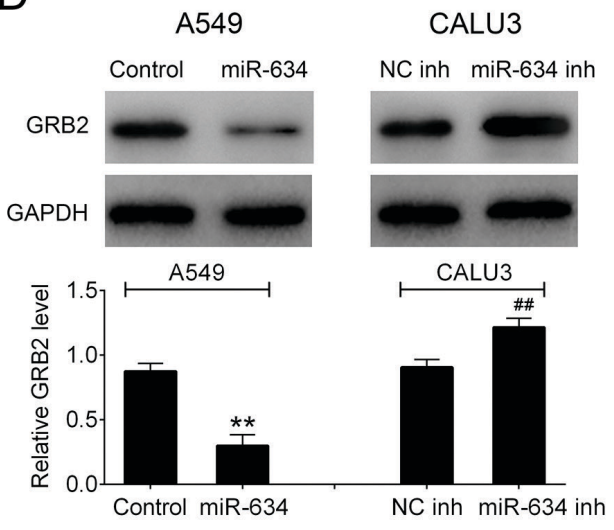

B

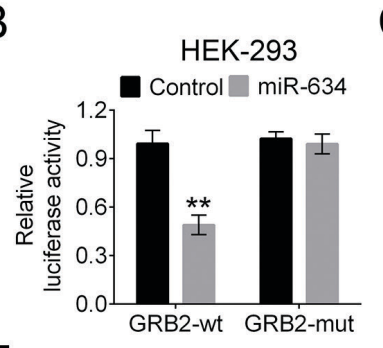

E

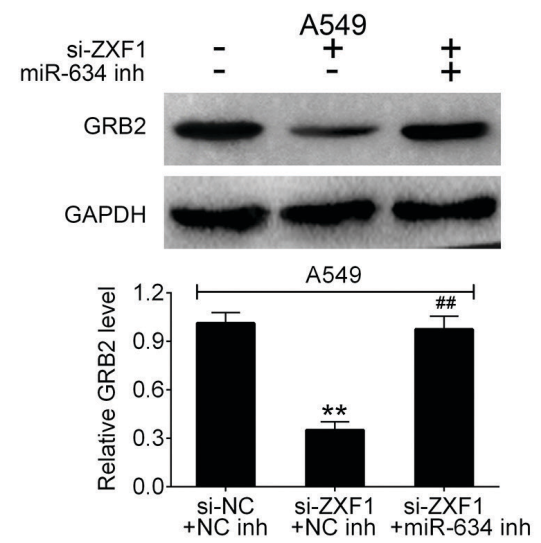

$+\mathrm{NC}$ inh +NC inh + miR-634 inh
CALU3

NC inh miR-634 inh

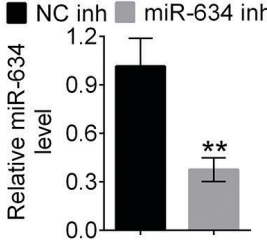

Figure 4. ZXF1 upregulated GRB2 expression by targeting miR-634 in vitro.

(A) The sequences of miR-634 binding sites complemented with GRB2 3'-UTR (362-368). GRB2-wt represented the entire 3'UTR sequences of wild type GRB2, GRB2-mut represented the mutated binding sites. (B) The binding affinity of miR-634 to GRB2-wt or its mutant were detected with luciferase reporter assay in HEK-293 cells. (C) The mRNA expression of miR-634 in miR-634 inhibitor-transfected CALU3 cells. (D) Western Blot was performed to detect the protein expression of GRB2 in miR-634 mimic-transfected A549 cells as well as NC inhibitor (NC-inh)-/miR-634 inhibitor(miR-634-inh)-transfected CALU3 cells. (E) The protein expression of GRB2 in A549 cells after transfection with si-ZXF1 or miR-634 inhibitor. Results are presented as mean \pm S.D. $p$ values lower than 0.05 indicated statistical significance. *Indicates $p<0.05, * *$ indicates $p<0.01$.

p21 in si-ZXF1-transfected LA cells. The data demonstrated that inhibition of ZXF1 resulted in a downregulation of Cyclin D1 and upregulation of p21. Moreover, co-transfection with GRB2 plasmid reversed the trend (Fig. 5E). This result indicated that overexpressing GRB2 could promote cell proliferation. Taken together, we found that ZXF1 increases LA cell proliferation by promoting GRB2 expression.

\section{ZXF1 promotes tumor growth of LA in vivo}

The above experiments explored the effect of ZXF1 on the proliferation of LA cells in vitro. Next, we tried to explore the in vivo function of ZXF1 on tumor proliferation using the $\mathrm{Balb} / \mathrm{c}$ nude mice xenograft model. Figure $6 \mathrm{~A}$ presents the photographs of tumors isolated 24 days after inoculation of stably transfected A549 cells into nude mice. The tumor size and volume were measured by caliper every 3 days (Fig. 6B). After 24 days the mice were sacrificed. The tumor weight was measured using analytical balance after 24 days (Fig. 6C). The results showed that the tumor size, weight and volume of sh-ZXF1-transfected xenograft tumor were decreased significantly in comparison to sh-NC-transfected xenograft tumor (control mice), indicating that ZXF1 inhibition enables inhibition of tumor growth in LA. Then, qRT-PCR was used to detect mRNA expression of ZXF1, miR-634 and GRB2 in sh-ZXF1-transfected and control mice. We found that sh-ZXF1 stably transfected xenograft tumor displayed a significantly downregulated expression of ZXF1 and GRB2 and upregulated miR-634 (Fig. 6D). Additionally, immunohistochemical staining analysis showed that isolated LA tumor tissues from nude mice with sh-ZXF1-transfected xenograft tumor implanted displayed lower expression of Ki-67 and GRB2 when compared to the control mice. This result strongly supported the finding that ZXF1 inhibition could suppress the tumor cell proliferation in vivo (Fig. 6E). Taken together, the in vivo studies demonstrated that ZXF1 knockdown could inhibit the tumor cell proliferation by targeting miR-634-GRB2 in vivo.

\section{DISCUSSION}

LA is one of the most serious malignant tumors with high recurrence and mortality rate. Despite great progress in traditional therapies, the overall five-year survival rate of patients with LA still remains unsatisfying (Qin et al., 2018). Therefore, to treat LA more efficiently and effectively, novel biomarkers and therapeutic methods need to be developed. Up to now, mounting evidence has been found that lncRNAs play important roles in the initiation and progression of LA, including lncRNA RGMB-AS1 (Li et al., 2016), lncRNA HOTAIR (Liu et al., 2013) and lncRNA CCAT2 (Qiu et al., 2014). Recently, Zhang et al found that overexpressed lncRNAZXF1 was associated with advanced tumor progression and poor prognosis in patients with LA. In addition, inhibition of ZXF1 decreased tumor cell proliferation (Zhang et al., 2014). ZXF1 might thus function in tumorigenesis and act as an important biomarker in LA progression. However, the molecular and biological functions as well as the targets of ZXF1 in the pathogenesis of LA are still not well investigated. 
A

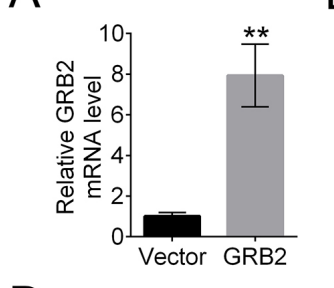

D

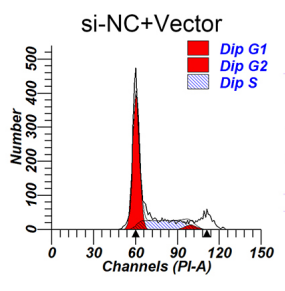

B
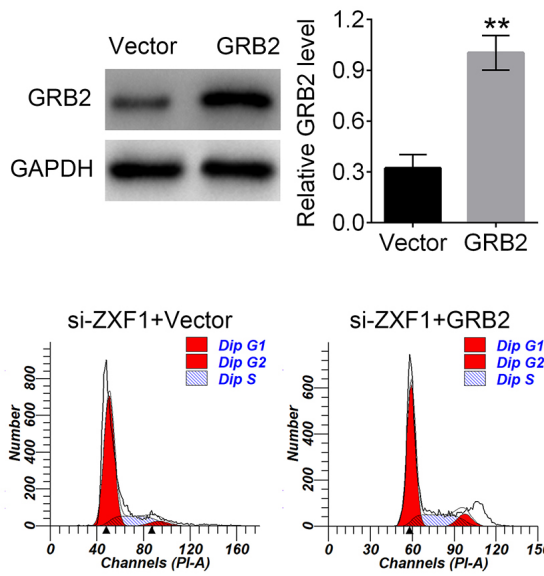

C
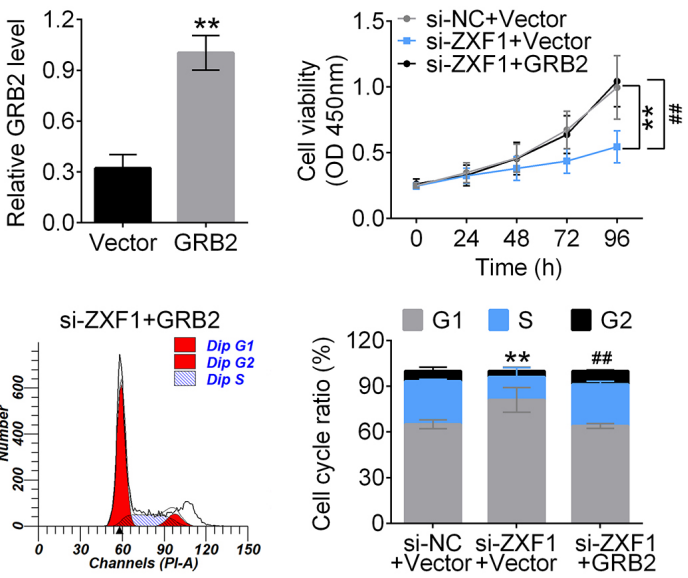

$E$
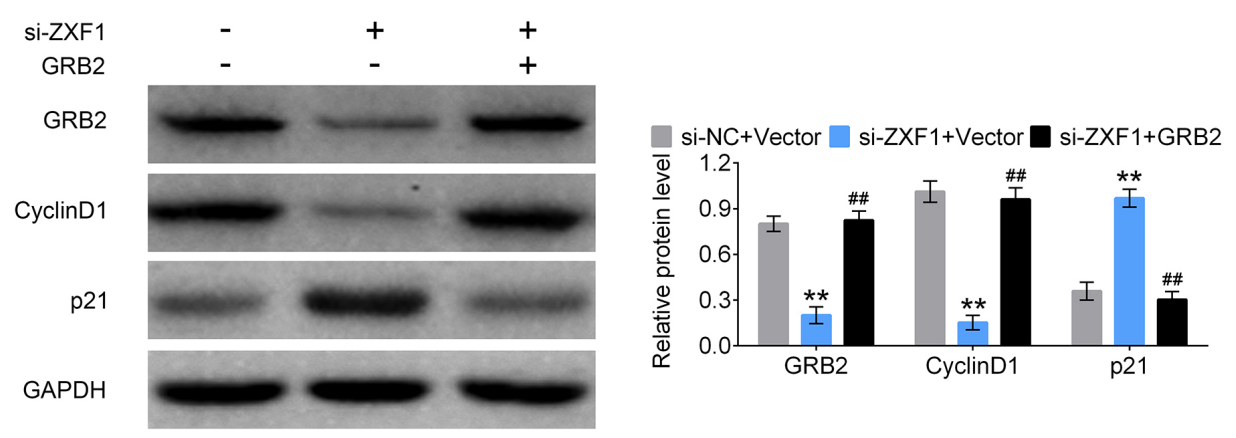

Figure 5. ZXF1 increased cell survival by promoting GRB2 expression.

(A) The mRNA expression of GRB2 in A549 cells after transfection with GRB2 plasmid determined by qRT-CPR. (B) The protein level of GRB2 in A549 cells after transfection with GRB2 plasmid determined by Western Blot. (C) The cell proliferation of A549 cells transfected with GRB2 plasmid and/or si-ZXF1 determined by CKK-8 assay. (D) The cell cycle phases distribution in GRB2 plasmid- and/or si-ZXF1transfected A549 cells was determined by flow cytometry analysis. (E) The protein levelsof CyclinD1 and p21 in GRB2 plasmid- and/or si-ZXF1-transfected A549 cells were detected by Western Blot. Dip denotes Diploid. Results are presented as mean \pm S.D. $p$ values lower than 0.05 indicated statistical significance. *Indicates $p<0.05, * *$ indicates $p<0.01$.

In this study, qRT-PCR assay showed that the expression level of ZXF1 was lower in tumor tissues from LA patients and related cancer cells. Moreover, survival rates analysis indicated that low-expression of ZXF1 could positively extend the survival time of patients with LA. We thus believe that ZXF1 might serve as an important biomarker for clinical diagnosis. Subsequently, the proliferation of LA cells (CALU3 and A549) was independently assessed by CCK-8 assay, flow cytometry analysis and Western Blot method. Taken together these in vitro results, we found that knockdown of ZXF1 inhibited LA cells growth and arrested the cell cycle in G1 phase. Besides, Western Blot assay indicated that inhibition of ZXF1 expression could increase p21 protein expression and decrease Cyclin D1 protein expression, which strongly suggests that ZXF1 serves as a cell cycle accelerator. Therefore, these data supported the hypothesis that ZXF1 promotes LA progression.

Many studies reported that lncRNAs serve as competing endogenous RNAs (ceRNAs), which could function as transcript sponges and inhibit miRNAs to attenuate their suppressive effect on target genes (Zhang et al., 2016). For example, lncRNA SNHG5 upregulated the expression of KLF4 by targeting miR-32 and promoted proliferation of gastric cancer cells (Zhao et al., 2017). A novel ceRNA, IncRNA HNF1A-AS1 mediates the inhibition of miR-34a-SIRT1-p53 axis to accelerate the progression of colon cancer (Fang et al., 2017). However, whether ZXF1 affects LA progression by regulat- ing miRNAs has not yet been studied. In this study, bioinformatics prediction and luciferase reporter assay revealed that miR-634 could directly bind to ZXF1 and act as an inhibitory target of ZXF1. In addition, RIP assay indicated that ZXF1 and miR-643 were higher in Anti-Ago2 group than that in Anti-IgG group. Furthermore, QRT-PCR assay showed that the mRNA expression of miR-634 was inhibited by ZXF1 overexpression in CALU3 cells. Conversely, knockdown of ZXF1 increased the miR-634 expression in A549 cells. We thus believe that miR-634 in LA cells is directly regulated by ZXF1.

GRB2, an important tumor metastasis regulatory protein, is involved in cell proliferation, cell motility, and contributes to cell cycle misregulation (Giubellino et al., 2008). Recently, mounting evidence showed that GRB2 acts as cancer-promoting gene in tumor initiation and progression of breast cancer, gastric cancer and lung cancer (Daly et al., 2002; Brown et al., 2008; Xu et al., 2011). It was also reported that GRB2 could act as a direct target of miR-634 and their interaction results in the suppression of growth and metastasis of ovarian cancer (van Jaarsveld et al., 2015). Thus, we verified whether ZXF1 regulates LA progression by affecting miR-634/GRB2 axis. Our data showed that ZXF1 positively regulated miR-634 expression in LA cells and ZXF1 modulated GRB2 expression by targeting miR-634 in vitro. Furthermore, we found that GRB2 was upregulated in LA cells and positively associated with the expression of ZXF1. 
A

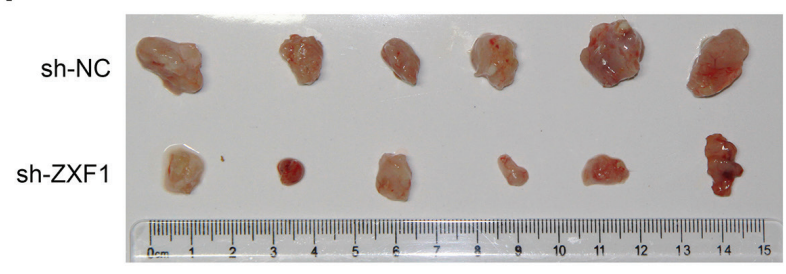

B

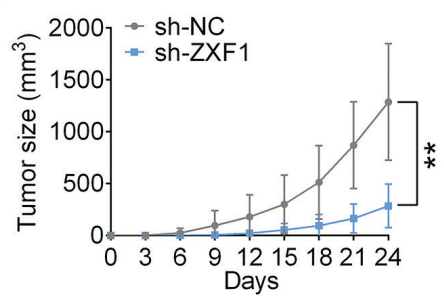

C
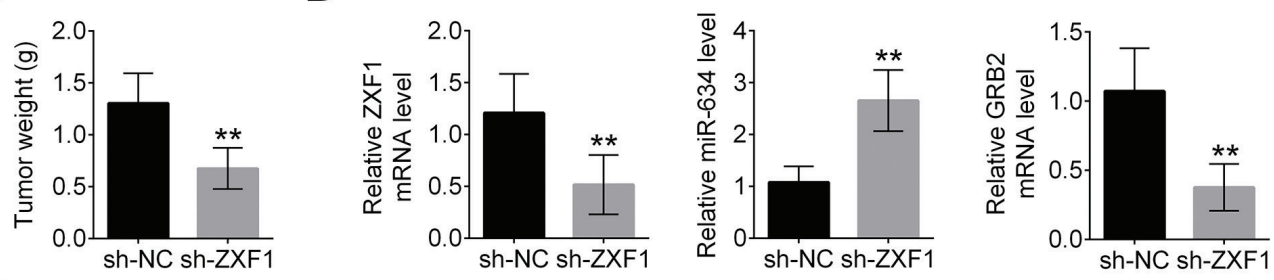

E
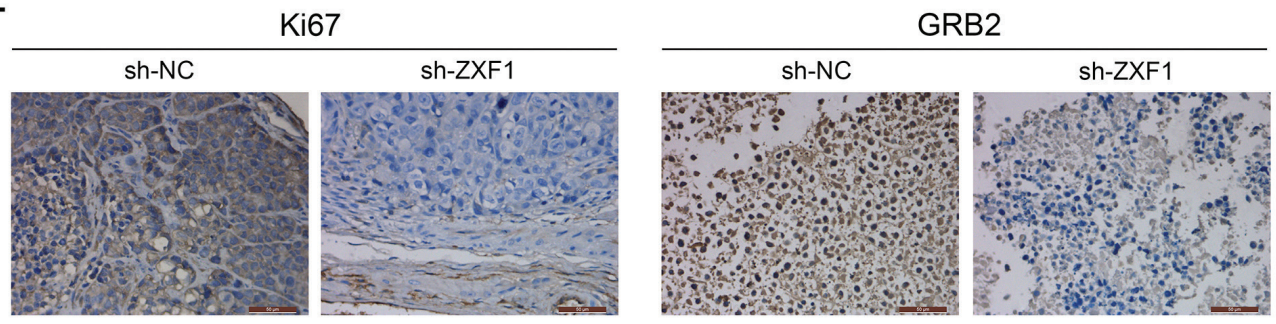

Figure 6. ZXF1 inhibition suppressed tumor growth in Balb/c nude mice xenograft model in vivo.

(A) Photographs of tumors isolated 24 days after inoculation of stably transfected A549 cells into nude mice. (B) Tumor volume measured by caliper every 3 days. (C) Tumor weight of the 2 groups of nude mice after 24 days. (D) The relative expression levels of ZXF1, miR-634 and GRB2 in tumor mouse model induced by A549 cells analyzed using qRT-PCR. (E) The effects of ZXF1 on the protein expressions of GRB2 and Ki67 in nude mouse lung cancer model induced by A549 cells determined based on immunohistochemical staining analysis. Results are presented as mean \pm SD. $p$ values lower than 0.05 indicated statistical significance. ${ }^{*}$ Indicates $p<0.05,{ }^{* *}$ indicates $p<0.01$.

Taken together, our data strongly demonstrated that ZXF1 promotes proliferation of LA cells by functioning as miR-634 sponge which indicates a novel ZXF1-miR634-GRB2 signaling pathway regulatory network in LA.

Specifically, this study showed that ZXF1 downregulates the expression of miR-634 and increases cell proliferation by upregulation of GRB2 expression. The molecular mechanism of ZXF1/miR-634/GRB2 axis in LA might provide a foundation for identifying new biomarkers for LA and new therapeutic targets for LA treatment.

\section{Competing interests}

The authors declare that they have no competing interests.

\section{Availability of data and materials}

All data generated or analyzed during this study are included in this published article.

\section{Authors' contribution}

YTZ conceived and designed the experiments, XBR analyzed and interpreted the results of the experiments, NX and TW performed the experiments

\section{Ethics approval and consent to participate}

The animal use protocol listed below has been reviewed and approved by the Animal Ethical and Welfare Committee.

\section{REFERENCES}

Bartel DP (2004) MicroRNAs: genomics, biogenesis, mechanism, and function. Cell 116: 281-297. https://doi.org/10.1016/s00928674(04)00045-5

Brown LA, SE Kalloger SE, Miller MA, Shih IeM, McKinney SE, Santos JL, Swenerton K, Spellman PT, Gray J, Gilks CB, Huntsman DG (2008) Amplification of 11q13 in ovarian carcinoma. Genes Chromosomes Cancer 47: 481-489. https://doi.org/10.1002/gcc.20549

Chen D, Si W, Shen J, Du C, Lou W, Bao C, Zheng H, Pan J, Zhong G, Xu L, Fu P, Fan W (2018) miR-27b-3p inhibits proliferation and potentially reverses multi-chemoresistance by targeting CBLB/ GRB2 in breast cancer cells. Cell Death Dis. 9: 188. https://doi. org/10.1038/s41419-017-0211-4

Cong J, Liu R, Wang X, Jiang H, Zhang Y (2016) MiR-634 decreases cell proliferation and induces apoptosis by targeting mTOR signaling pathway in cervical cancer cells. Artif Cells Nanomed Biotechnol 44: 1694-1701. https://doi.org/10.3109/21691401.2015.1080171

Daly RJ, Gu H, Parmar J, Malaney S, Lyons RJ, Kairouz R, Head DR, Henshall SM, Neel BG, Sutherland RL (2002) The docking protein Gab2 is overexpressed and estrogen regulated in human breast cancer. Oncogene 21: 5175-5181. https://doi.org/10.1038/sj.onc.1205522

Dickson I (2016) Hepatocellular carcinoma: A role for lncRNA in liver cancer. Nat Rev Gastroenterol Hepatol 13: 122-123. https://doi. org/10.1038/nrgastro.2016.21

Fang C, Qiu S, Sun F, Li W, Wang Z, Yue B, Wu X, Yan D (2017) Long non-coding RNA HNF1A-AS1 mediated repression of miR34a/SIRT1/p53 feedback loop promotes the metastatic progression of colon cancer by functioning as a competing endogenous RNA. Cancer Lett 410: 50-62. https:// doi.org/10.1016/j.canlet.2017.09.012

Fujiwara N, Inoue J, Kawano T, Tanimoto K, Kozaki K, Inazawa J (2015) miR-634 Activates the mitochondrial apoptosis pathway and enhances chemotherapy-induced cytotoxicity. Cancer Res 75: 38903901. https://doi.org/10.1158/0008-5472.CAN-15-0257

Giubellino A, Burke TR Jr, Bottaro DP (2008) Grb2 signaling in cell motility and cancer. Expert Opin Ther Targets 12: 1021-1033. https:// doi.org/10.1517/14728222.12.8.1021

Jiang W, Wei K, Pan C, Li H, Cao J, Han X, Tang Y, Zhu S, Yuan W, He Y, Xia Y, Chen L, Chen Y (2018) MicroRNA-1258 suppresses 
tumour progression via GRB2/Ras/Erk pathway in non-smallcell lung cancer. Cell Prolif 51: e12502. https://doi.org/10.1111/ cpr. 12502

Li LY, Li EM, Wu ZY, Cao HH, Shen JH, Xu XE, Chen B, Wu JY, Xu LY (2014) Overexpression of GRB2 is correlated with lymph node metastasis and poor prognosis in esophageal squamous cell carcinoma. Int J Clin Exp Pathol 7: 3132-3140. PMCID: PMC4097250

Li P, Zhang G, Li J, Yang R, Chen S, Wu S, Zhang F, Bai Y, Zhao H, Wang Y, Dun S, Chen X, Sun Q, Zhao G (2016) Long Noncoding RNA RGMB-AS1 Indicates a Poor Prognosis and Modulates Cell Proliferation, Migration and Invasion in Lung Adenocarcinoma. PLoS One 11: e0150790. https://doi.org/10.1371/journal. pone.0150790

Liu Z, Sun M, Lu K, Liu J, Zhang M, Wu W, De W, Wang Z, Wang $\mathrm{R}$ (2013) The long noncoding RNA HOTAIR contributes to cisplatin resistance of human lung adenocarcinoma cells via downregualtion of p21(WAF1/CIP1) expression. PLoS One 8: e77293. https:// doi.org/10.1371/journal.pone.0077293

Lowenstein EJ, Daly RJ, Batzer AG, Li W, Margolis B, Lammers R, Ullrich A, Skolnik EY, Bar-Sagi D, Schlessinger J (1992) The SH2 and SH3 domain-containing protein GRB2 links receptor tyrosine kinases to ras signaling. Cell 70: 431-442. https://doi. org/10.1016/0092-8674(92)90167-b

Ni X, Liao Y, Li L, Zhang X, Wu Z (2018) Therapeutic role of long non-coding RNA TCONS_00019174 in depressive disorders is dependent on Wnt/beta-catenin signaling pathway. I Integr Neurosci 17: 125-132. https://doi.org/10.31083/JIN-170052

Nikpayam E, Tasharrofi B, Sarrafzadeh S, Ghafouri-Fard S (2017) The role of long non-coding RNAs in ovarian cancer. Iran Biomed J 21: 3-15. https://doi.org/10.6091/.21.1.24

Pan GF, Zhou XF, Zhao JP (2017) Correlation between expression of long non-coding RNA ZXF1 and prognosis of lung adenocarcinoma and its potential molecular mechanism. Zhonghua Zhong Liu Za Zhi 39: 102-108. https://doi.org/10.3760/cmaj.issn.0253-3766.2017.02.006

Ponting CP, Oliver PL, Reik W (2009) Evolution and functions of long noncoding RNAs. Cell 136: 629-641. https://doi.org/10.1016/i. cell.2009.02.006

Qin H, Wang F, Liu H, Zeng Z, Wang S, Pan X, Gao H (2018) New advances in immunotherapy for non-small cell lung cancer. $\mathrm{Am} J$ Transl Res 10: 2234-2245. PMCID: PMC6129543

Qiu M, Xu Y, Yang X, Wang J, Hu J, Xu L, Yin R (2014) CCAT2 is a lung adenocarcinoma-specific long non-coding RNA and promotes invasion of non-small cell lung cancer. Tumour Biol 35: 5375-5380. https://doi.org/10.1007/s13277-014-1700-z

Shi Q, Wang Y, Mu Y, Wang X, Fan Q (2018) MiR-433-3p inhibits proliferation and invasion of esophageal squamous cell carcinoma by targeting GRB2. Cell Physiol Biochem 46: 2187-2196. https://doi. org/10.1159/000489548
Siegel RL, Miller KD, Jemal A (2018) Cancer statistics, 2018. CA Cancer J Clin 68: 7-30. https://doi.org/10.3322/caac.21442

van Jaarsveld MT, van Kuijk PF, Boersma AW, Helleman J, van IJcken WF, Mathijssen RH, Pothof J, Berns EM, Verweij J, Wiemer EA (2015) miR-634 restores drug sensitivity in resistant ovarian cancer cells by targeting the Ras-MAPK pathway. Mol Cancer 14: 196. https://doi.org/10.1186/s12943-015-0464-4

Wakeam E, Acuna SA, Leighl NB, Giuliani ME, Finlayson SRG, Varghese TK, Darling GE (2017) Surgery versus chemotherapy and radiotherapy for early and locally advanced small cell lung cancer: a propensity-matched analysis of survival. Lung Cancer 109: 78-88. https://doi.org/10.1016/j.lungcan.2017.04.021

Wang X, Lu X, Zhang T, Wen C, Shi M, Tang X, Chen H, Peng C, Li H, Fang Y, Deng X, Shen B (2016) mir-329 Restricts tumor growth by targeting grb2 in pancreatic cancer. Oncotarget 7: 21441-21453. https://doi.org/10.18632/oncotarget.7375

Xu XL, Wang X, Chen ZL, Jin M, Yang W, Zhao GF, Li JW (2011) Overexpression of Grb2-associated binder 2 in human lung cancer. Int J Biol Sci 7: 496-504. https://doi.org/10.7150/ijbs.7.496

Yu GZ, Chen Y, Wang JJ (2009) Overexpression of Grb2/HER2 signaling in Chinese gastric cancer: their relationship with clinicopathological parameters and prognostic significance. I Cancer Res Clin Oncol 135: 1331-1339. https://doi.org/10.1007/s00432-009-0574-8

Zappa C, Mousa SA (2016) Non-small cell lung cancer: current treatment and future advances. Transl Lung Cancer Res 5: 288-300. https://doi.org/10.21037/tlcr.2016.06.07

Zhan Y, Zang H, Feng J, Lu J, Chen L, Fan S (2017) Long non-coding RNAs associated with non-small cell lung cancer. Oncotarget 8: 69174-69184. https://doi.org/10.18632/oncotarget.20088

Cao Y1, Fu J, Yun JP, Zhang MF, Zhang CZ (2016) miR-634 exhibits anti-tumor activities toward hepatocellular carcinoma via Rab1A and DHX33. Mol Oncol 10: 1532-1541. https://doi.org/10.1016/j. molonc.2016.09.001

Zhang K, Li Q, Kang X, Wang Y, Wang S (2016) Identification and functional characterization of lncRNAs acting as ceRNA involved in the malignant progression of glioblastoma multiforme. Oncol Rep 36: 2911-2925. https://doi.org/10.3892/or.2016.5070

Zhang L, Zhou XF, Pan GF, Zhao JP (2014) Enhanced expression of long non-coding RNA ZXF1 promoted the invasion and metastasis in lung adenocarcinoma. Biomed Pharmacother 68: 401-407. https:// doi.org/10.1016/j.biopha.2014.03.001

Zhao L, Han T, Li Y, Sun J, Zhang S, Liu Y, Shan B, Zheng D, Shi J (2017) The lncRNA SNHG5/miR-32 axis regulates gastric cancer cell proliferation and migration by targeting KLF4. FASEB J 31: 893-903. https://doi.org/10.1096/fj.201600994R

Zhou S, He Y, Yang S, Hu J, Zhang Q, Chen W, Xu H, Zhang H, Zhong S, Zhao J, Tang J (2018) The regulatory roles of lncRNAs in the process of breast cancer invasion and metastasis. Biosci Rep. 38: https://doi.org/10.1042/BSR20180772 